\title{
Non-singular screw dislocations as the Coulomb gas with smoothed out coupling and the renormalization of the shear modulus*
}

\author{
C. Malyshev \\ Steklov Institute of Mathematics (St.-Petersburg Department) \\ Fontanka 27, St.-Petersburg, 191023, RUSSIA
}

\begin{abstract}
A field theory is developed for a thermodynamical description of an array of parallel non-singular screw dislocations in an elastic cylinder. The partition function of the system is considered in the functional integral form. Self-energy of the dislocation cores is chosen in the form suggested by the gauge-translational model of non-singular screw dislocation. It is shown that the system of the dislocations is equivalent to the two-dimensional Coulomb gas. The coupling potential is prevented from a short-distance divergency since the core energies are taken into account. Twopoint correlation functions of the stress components are obtained. Renormalization of the shear modulus caused by the presence of the dislocations is studied in the approximation of non-interacting dislocation dipoles. It is demonstrated that the finite size of the dislocation cores results in a modification of the renormalization law.
\end{abstract}

Key words: functional integration, dislocation, Coulomb gas, renormalization

*Presented at the International Workshop "Classical and Quantum Integrable Systems (CQIS-2011)" (January 24-27, 2011, Protvino, Russian Federation) 


\section{Introduction}

Non-trivial topologically configurations in ordered states (e.g. vortices, dislocations, and other defects) attract appreciable attention in modern condensed matter physics. For instance, dislocations as imperfections of the crystalline ordering are of importance for structural, transport, and electronic properties of real solids. Two-dimensional ordered states are of special interest due to the significance of the topological defects for the corresponding phase transitions [1-6]. The ideas of [1-6] have been further elaborated for the description of the dislocation-mediated crystal melting in two dimensions [7-11]. The textbooks [12,13] summarize an original approach to the ordered states and phase transitions dominated by the line-like disturbances. The peculiarity of [12,13] is due to a systematical usage of (singular) gauge fields. Certain aspects of the statistical physics of dislocations are considered in [14-18].

Dislocations have recently attracted considerable attention as far as the physics of the nanotubes is concerned [19-22. The point is that multilayer nanotubes can contain within their walls screw dislocations lying along the axis of the tube [21]. The external diameter of the nanotubes ranges from nanometers to tens of nanometers. The wall thickness varies from one to several tens of atomic layers. Therefore, effects influenced by the dislocation cores look attractive for study in the context of the nanotubes since the core sizes are comparable with those of atomic layers. Note that the electronic and mechanical properties of the graphene sheets in presence of dislocations are also of interest [23 25].

According to the elasticity theory, the stress tensor components of a single dislocation are singular on the defect line. In reality, the stress components are smoothed out because of the formation of the core regions. Since the first attempts [26, 27], various approaches to dislocations with non-trivial core are known. For instance, the quasi-continuum approach [28, 29], the gradient elasticity [30,31], and the Lagrangian translational gauging [33 37] enable one to obtain appropriate solutions for non-singular screw dislocations.

The elastic stresses of the screw dislocation are obtained in [33 37] in the form of the superposition of the conventional far-reaching ("background") contribution and a shortranged ("gauge") correction. The latter modifies the background fields within a compact core. Therefore, smoothing of the singular behaviour of the conventional screw dislocation occurs thus leading to the so-called modified screw dislocation. Note that the secondorder elasticity is also of importance for the defects in crystals [38. The approach of [35] is suitable for study of the second order stresses in the case of a non-singular screw dislocation [39].

This paper is concerned with a thermodynamical description of a large number of the modified screw dislocations [35] lying within a long enough cylinder of a circular crosssection. The functional integration [40 45] is used to represent the partition function and to study certain averages. The core energies are accounted for in accordance with [35]. It is shown that the collection of parallel non-singular dislocations is equivalent to the two-dimensional Coulomb-like system of charges interacting via the potential which is logarithmic at large distances but vanishes locally (smoothed out coupling). The twodimensional Coulomb gas [46] belongs to the class of systems covered by the theory [1-6], and it demonstrates a relationship with the two-dimensional spin models [47 49].

Dislocations available in a solid result in the renormalization of the corresponding

elastic moduli [9, 16]. Two-point correlation functions of the stress components are calculated in this paper for investigating of the renormalization of the shear modulus. It 
is demonstrated that non-triviality of the cores results in a correction to the law of the renormalization of the shear modulus what could be appreciable for the nanotubes. The present field-theoretical approach is influenced technically by that of [50,51], where certain correlation functions have been calculated for the string models possessing the world-sheet vortices.

The paper is organized as follows. section 1 is the introductory section. The partition function of the elastic cylinder containing the array of the modified screw dislocations is considered in section 2, Transformation of collection of the dislocations to a dual system of two-dimensional charges is presented in section 2. In section 3, we calculate certain thermodynamical averages, e.g., the average square of the dislocation dipole momentum and the stress-stress correlation function in the approximation of non-interacting dislocation dipoles. The renormalization of the shear modulus in the presence of non-singular dislocations is studied section 4. Discussion in section 5 closes the paper.

\section{The partition function}

Consider a circular cylinder containing straight non-singular screw dislocations lying along the axis of the cylinder. The cylinder's material is approximated by elastically-isotropic continuum described by linear elasticity. Recall that the screw dislocation is characterized by the parallelism between its Burgers vector and tangent to the dislocation line [52,53]. The modified screw dislocation [35] is a point of departure of the present investigation that treats the distribution of the dislocations as a thermodynamical ensemble at nonzero temperature. The functional integration approach is used below to investigate the partition function and certain correlation functions. In what follows, the Cartesian axis $O x_{3}$ is along the cylinder's axis.

Let us begin with the functional integral representation of the thermodynamical partition function $\mathcal{Z}$ of the elastic cylinder containing the non-singular dislocations:

$$
\begin{aligned}
& \mathcal{Z}=\frac{1}{N} \int e^{-\beta W} \mathcal{D}\left(\sigma_{i j}^{\mathrm{b}}, \sigma_{i j}^{\mathrm{c}}, u_{i}, e_{i j}\right) \\
& W \equiv E-i E_{\text {ext }}, \quad E \equiv E_{\text {el }}+E_{\text {core }}
\end{aligned}
$$

where $\beta$ is inverse of the absolute temperature $T$ (the Boltzmann constant is unity). The functional $W$ (2) consists of the following contributions (indices repeated imply summation):

$$
\begin{aligned}
& E_{\text {el }}=\frac{1}{4 \mu} \int\left(\left(\sigma_{i j}^{\mathrm{b}}+\sigma_{i j}^{\mathrm{c}}\right)^{2}-\frac{\nu}{1+\nu}\left(\sigma_{i i}^{\mathrm{b}}+\sigma_{i i}^{\mathrm{c}}\right)^{2}\right) \mathrm{d}^{3} x, \\
& E_{\text {core }}=\int\left(\ell e_{i j}(\text { inc } e)_{i j}-e_{i j} \sigma_{i j}^{\mathrm{c}}\right) \mathrm{d}^{3} x, \\
& E_{\text {ext }}=\frac{1}{2} \int \sigma_{i j}^{\mathrm{b}}\left(\partial_{i} u_{j}+\partial_{j} u_{i}-2 \mathcal{P}_{i j}\right) \mathrm{d}^{3} x,
\end{aligned}
$$

where (inc $e)_{i j}$ denotes the double-curl operator: $-\epsilon_{i k l} \epsilon_{j m n} \partial_{k} \partial_{m} e_{l n}\left(\epsilon_{i k l}\right.$ is totally antisymmetric tensor). The functional $E_{\mathrm{el}}(3)$ is the elastic energy of superposition of two stresses, $\sigma_{i j}^{\mathrm{b}}$ and $\sigma_{i j}^{\mathrm{c}}$, provided $\mu$ and $\nu$ are respectively identified as the shear modulus and the Poisson ratio. The notation $\sigma_{i j}^{\mathrm{b}}$ is reserved for the long-ranged contribution, while $\sigma_{i j}^{\mathrm{c}}$ describes a non-conventional stress which modifies the background one, $\sigma_{i j}^{\mathrm{b}}$, within the core 
of the modified dislocation [35, 11. The dislocation core energy is given by $E_{\text {core }}$ (3), where $e_{i j}$ (inc $\left.e\right)_{i j}$ originates (by means of linearizations) from the Hilbert-Einstein Lagrangian proposed in [35] in the framework of the translational gauge approach to dislocations 2 . Here $e_{i j}$ is the total strain tensor, and $\ell$ is a scale of the dislocation core energy. The term $E_{\text {ext }}$ (3) is linear with respect to (symmetrized) derivatives of the displacement vector $u_{i}$, as well as with respect to a "source" $\mathcal{P}_{i j}$ which is related to the plastic strain $e_{i j}^{P}$ as follows: $\mathcal{P}_{i j}=e_{i j}^{P}+C_{i j}$. The specific configuration of singular dislocation lines is prescribed by appropriately chosen $e_{i j}^{P}$ since the plastic strain is concentrated on cut surfaces bounded by the dislocation lines [59,60]. The present approach enables one to avoid the stress divergencies on the dislocation lines. In this respect, an auxiliary field $C_{i j}$ (which is not a variable of the functional integration) is postulated to ensure the background fixing at $\ell \neq 0$ in such way that the components of $\sigma_{i j}^{\mathrm{b}}$ just coincide with the conventional dislocation stresses governed by $e_{i j}^{P}\left(C_{i j}\right.$ vanishes at $\left.\ell=0\right)$.

The notation $\mathcal{D}\left(\sigma_{i j}^{\mathrm{b}}, \sigma_{i j}^{\mathrm{c}}, u_{i}, e_{i j}\right)$ implies the integration measure in (11) equal to a product of particular measures for each functional variable marked inside the brackets. More details on the definition of the functional integration measure by means of the appropriate limiting procedure can be found in [40 45]. The normalization factor $1 / N$ is to absorb physically irrelevant multiplicative infinities. The partition function given by (1), (2) naturally generalizes the partition function of the array of singular dislocations [12, 13]. We shall apply the steepest descent approximation [40 45] to $\mathcal{Z}$ given by (11), (2). The corresponding saddle point solutions are determined from requirement that the variation of the functional $W$ vanishes, $\delta W=0$, under independent variations of its functional arguments.

Firstly, the following observation concerning $\mathcal{Z}(\mathbb{1})$ is in order provided three-dimensional Eqs. (3) are viewed at $\ell=0$. Shifting subsequently $\sigma_{i j}^{\mathrm{c}} \rightarrow \sigma_{i j}^{\mathrm{c}}+\hat{\sigma}_{i j}$ and $e_{i j} \rightarrow e_{i j}+\hat{e}_{i j}$ (here, $\hat{\sigma}_{i j}$ and $\hat{e}_{i j}$ are new adjustable fields), one can remove in $E$ the terms linear in $\sigma_{i j}^{\mathrm{c}}$ and $e_{i j}$. Then, the integrations over $\sigma_{i j}^{\mathrm{c}}$ and $e_{i j}$ are decoupled and can be compensated by redefinition of $1 / N$. Eventually, $E$ is reduced to $E_{\mathrm{el}}=E_{\mathrm{el}}\left(\sigma_{i j}^{\mathrm{b}}\right)$, and two remaining integrations, over $u$ and $\sigma_{i j}^{\mathrm{b}}$, result in the partition function of the array of singular dislocations characterized by a specific choice of $e_{i j}^{P}[12,13]$.

Our framework is that of the plane elasticity since it is assumed that the cylinder is long enough [52,53]. The independence on the third coordinate reduces our study to the two dimensional problem. Therefore, the functionals (3) take the form:

$$
\begin{aligned}
& E_{\text {el }}=\frac{1}{2 \mu} \int\left(\sigma_{i}^{\mathrm{b}}+\sigma_{i}^{\mathrm{c}}\right)^{2} \mathrm{~d}^{2} x, \\
& E_{\text {core }}=2 \int\left(\ell e_{i}(\text { inc } e)_{i}-e_{i} \sigma_{i}^{\mathrm{c}}\right) \mathrm{d}^{2} x, \\
& E_{\text {ext }}=\int \sigma_{i}^{\mathrm{b}}\left(\partial_{i} u-2 \mathcal{P}_{i}\right) \mathrm{d}^{2} x,
\end{aligned}
$$

where the integrands are $\left(x_{1}, x_{2}\right)$-dependent, and summation goes over $i=1,2$. Since the displacement vector of the straight screw dislocation is along $O x_{3}$ [52], we use $u \equiv u_{3}$. In

\footnotetext{
${ }^{1}$ Two stress fields $\sigma_{i j}^{\mathrm{b}}$ and $\sigma_{i j}^{\mathrm{c}}$ correspond to splitting of the total elastic stress into, so-called, "background" and "gauge" parts [34 37].

${ }^{2}$ The gauge Lagrangians quadratic in the dislocation densities are used in [34,36] what is equivalent, in the case of the screw dislocation, to the approach of [35]. The most general three-dimensional gauge Lagrangian has been discussed in [54]. Certain developments in the gauge gravity [13, 55, 58] involve the gauge-translational features of the theory of dislocations.
} 
addition, we abbreviate: $\sigma_{i}^{\#} \equiv \sigma_{i 3}^{\#}$ (\# is b or c), $e_{i} \equiv e_{i 3}$, etc. For instance, the notation for the functional integration measure is of the form: $\mathcal{D}\left(\sigma_{i}^{\mathrm{b}}, \sigma_{i}^{\mathrm{c}}, u, e_{i}\right)$.

Let us consider equations (4) at $\ell=0$. The source $\mathcal{P}_{i}$ is taken at $\ell=0$ in the form: $\mathcal{P}_{i}=e_{i}^{P}$. A single positively oriented straight screw dislocation intersecting the plane $x_{1} O x_{2}$ at $\mathbf{x}=\mathbf{y}$ (we use $\mathbf{x} \equiv\left(x_{1}, x_{2}\right)$ ) is "produced" by the plastic strain with a single non-zero component $e_{2}^{P}=(b / 2) \theta\left(-x_{1}+y_{1}\right) \delta\left(y_{2}-x_{2}\right)$ [59,60]. Here, $\theta(\cdot)$ is the Heaviside function and $b$ is absolute value of the Burgers vector $\mathbf{b}$ lying along $O x_{3}$. Furthermore, the integration over $u$ is equivalent to the insertion of the delta-functional $\delta\left(\partial_{i} \sigma_{i}^{\mathrm{b}}\right)$. The equilibrium equation $\partial_{i} \sigma_{i}^{\mathrm{b}}=0$ is fulfilled by the Kröner ansatz $\sigma_{i}^{\mathrm{b}} \equiv \mu \epsilon_{i j} \partial_{j} f^{\mathrm{b}}$ (see [61]), where $\epsilon_{i j}$ is totally antisymmetric symbol of second rank. Vanishing of the variation $\delta W$ under the shift $f^{\mathrm{b}} \rightarrow f^{\mathrm{b}}+\delta f^{\mathrm{b}}$ allows one to determine the potential $f^{\mathrm{b}}$.

Consider a collection of $\mathcal{N}$ screw dislocations with the coordinates $\mathbf{x}=\mathbf{y}_{I}$ and the Burgers vectors $\mathbf{b}_{I}, 1 \leq I \leq \mathcal{N}$. A disc of radius $R$ is a cross-section of the cylinder containing the dislocations. Assume that the defect positions on the disc are well separated from each other as well as from the boundary (the distribution of the dislocations is not too dense). For our purposes it is appropriate to consider the solution corresponding to the infinite plane, i.e., the influence of the boundary is neglected at a large enough $R$. Taking into account arbitrariness of $\delta f^{\mathrm{b}}$, we obtain the extremum condition:

$$
\Delta f^{\mathrm{b}}=2 i\left(\partial_{1} e_{2}^{P}-\partial_{2} e_{1}^{P}\right)=-i \rho(\mathbf{x}), \quad \rho(\mathbf{x}) \equiv \sum_{I=1}^{\mathcal{N}} j\left(\mathbf{x}-\mathbf{y}_{I}\right),
$$

where $j\left(\mathbf{x}-\mathbf{y}_{I}\right) \equiv b_{I} \stackrel{(2)}{\delta}\left(\mathbf{x}-\mathbf{y}_{I}\right) \stackrel{(2)}{\delta}(\mathbf{x})$ is the delta-function on $x_{1} O x_{2}$, and $\rho(\mathbf{x})$ is thus the dislocation density. We determine $f^{\mathrm{b}}$ from (5) using the Prandtl stress potential [61] for the collection of the screw dislocations:

$$
f^{\mathrm{b}}=\frac{-i}{2 \pi} \sum_{I=1}^{\mathcal{N}} b_{I} \log \left|\mathbf{x}-\mathbf{y}_{I}\right| .
$$

Now we turn to $\ell \neq 0$ and require vanishing of $\delta W$ for independent variations of the functional arguments of $W$. Generally, provided that Eqs. (3) are considered, the variation of $\sigma_{i j}^{\mathrm{c}}$ results in the 'strain-stress' constitutive law:

$$
e_{i j}=\frac{1}{2 \mu}\left(\sigma_{i j}^{\mathrm{b}}+\sigma_{i j}^{\mathrm{c}}-\frac{\nu}{1+\nu}\left(\sigma_{l l}^{\mathrm{b}}+\sigma_{l l}^{\mathrm{c}}\right) \delta_{i j}\right) .
$$

Also, the variation of $e_{i j}$ gives the equation:

$$
(\text { inc } e)_{i j}=\frac{1}{2 \ell} \sigma_{i j}^{\mathrm{c}}
$$

which is a linearized version of the Einstein-type gauge equation investigated in the nonlinear approach of [39]. Since $\partial_{i}(\text { inc } e)_{i j} \equiv 0$, we put (in the plane problem) $\sigma_{i}^{\mathrm{c}}=\mu \epsilon_{i j} \partial_{j} f^{\mathrm{c}}$ to fulfil $\partial_{i} \sigma_{i}^{\mathrm{c}}=0$. Arbitrariness of the variations $\delta f^{\mathrm{b}}$ and $\delta f^{\mathrm{c}}$ results in the extremum conditions:

$$
\begin{aligned}
& \Delta\left(f^{\mathrm{b}}+f^{\mathrm{c}}\right)=2 i\left(\partial_{1} \mathcal{P}_{2}-\partial_{2} \mathcal{P}_{1}\right), \\
& \Delta\left(f^{\mathrm{b}}+f^{\mathrm{c}}\right)=\kappa^{2} f^{\mathrm{c}}, \quad \kappa^{2} \equiv \frac{\mu}{\ell},
\end{aligned}
$$


where $\mathcal{P}_{i} \equiv e_{i}^{P}+C_{i}$, and Eqs. (5) -(8) are accounted for. Self-consistency of Eqs. (9) requires coincidence of their right-hand sides and takes the form:

$$
\partial_{1} C_{2}-\partial_{2} C_{1}=\frac{1}{2}\left(\rho-i \kappa^{2} f^{\mathrm{c}}\right)
$$

where $\rho \equiv \rho(\mathbf{x})$ is defined in (5) We represent a general solution to (10) as follows:

$$
C_{i}=\frac{i}{2}\left(\partial_{i} \phi+\epsilon_{i j} \partial_{j} \psi\right)
$$

where $\phi$ is the arbitrary regular function, while $\psi$ is to be found after substitution of (11) to (10). If (10) is respected, either $f^{\mathrm{b}}$ or $f^{\mathrm{c}}$ can be fixed arbitrarily. Our strategy at $\ell \neq 0$ is to keep $f^{\mathrm{b}}$ still respecting Eq. (5). Then Eqs. (9) lead to a single equation for $f^{\mathrm{c}}$ :

$$
\left(\Delta-\kappa^{2}\right) f^{\mathrm{c}}=i \rho(\mathbf{x}),
$$

which is solved as follows:

$$
f^{\mathrm{c}}=\frac{-i}{2 \pi} \sum_{I=1}^{\mathcal{N}} b_{I} K_{0}\left(\kappa\left|\mathbf{x}-\mathbf{y}_{I}\right|\right),
$$

where $K_{0}(\cdot)$ is the modified Bessel function. The choice $\phi=0, \psi=f^{\mathrm{c}}$ ensures coincidence of (10) and (12).

Therefore, $\frac{1}{i}\left(f^{\mathrm{b}}+f^{\mathrm{c}}\right)$ is the stress potential of the collection of non-singular screw dislocations. The fields $\sigma_{i}^{\mathrm{b}}$ and $\sigma_{i}^{\mathrm{c}}$ are rather the saddle point solutions of the functional $W$ (21), while the proper stress fields are given by $\frac{1}{i} \sigma_{i}^{\mathrm{b}}$ and $\frac{1}{i} \sigma_{i}^{\mathrm{c}}$. Equations (6) and (13) demonstrate that a modification of the Prandtl stress potential $\frac{1}{i} f^{\mathrm{b}}$ occurs within the cores, i.e., within the tubular vicinities of transverse size $\simeq \kappa^{-1}$. When $\kappa\left|\mathbf{x}-\mathbf{y}_{I}\right| \gg 1, \forall I$, the total solution is dominated by the conventional contribution $\frac{1}{i} f^{\mathrm{b}}$. The sum $\frac{1}{i}\left(f^{\mathrm{b}}+f^{\mathrm{c}}\right)$ behaves smoothly when $\mathbf{x}$ is close to either of $\mathbf{y}_{I}$, and the known singularities in the total stress distribution $\frac{1}{i}\left(\sigma_{i}^{\mathrm{b}}+\sigma_{i}^{\mathrm{c}}\right)$ do not appear.

Thus, the stationary solution corresponds to the modified screw dislocation [35] which is, in turn, in agreement with the solution obtained by means of the gradient elasticity [31, 32]. The gradient elasticity itself belongs to a class of the generalized continuum theories which effectively take into account interatomic forces for the explanation of the material behaviour on the nano-scales (and thus inside the defect cores) 62. The solution obtained will be used to estimate the partition function given by (11), (2) and (4) using the steepest descent approximation.

Let us proceed with the consideration of the partition function $\mathcal{Z}$. Integration by parts in $W$ transforms $e_{i}(\text { inc } e)_{i}$ into the quadratic expression, $-\frac{1}{2}\left(\partial_{i} e_{j}-\partial_{j} e_{i}\right)^{2}$, invariant under the shift $e_{i} \rightarrow e_{i}+\partial_{i} g$, where $g$ is arbitrary function (the Abelian gauge transformation). To restrict the gauge arbitrariness related to the functional integration over $e_{i}$, let us impose, so-called, "Coulomb gauge" $\partial_{i} e_{i}=0$ [40]. Finally, the contribution $\ell e_{i}(\text { inc } e)_{i}$ in $W$ is replaced by $\ell e_{i} \Delta e_{i}$, and the shift

$$
e_{i} \rightarrow e_{i}+(2 \ell)^{-1} \Delta^{-1} \sigma_{i}^{\mathrm{c}}
$$

cancels the term linear in $e_{i}$. The Green function of two-dimensional Laplacian $\Delta^{-1}$ acts as convolution operator. The resulting Gaussian integration over $e_{i}$,

$$
\int e^{-2 \beta \ell \int e_{i} \Delta e_{i} \mathrm{~d}^{2} x} \mathcal{D}\left(e_{i}\right)
$$


is absorbed into $1 / N$, and the partition function $\mathcal{Z}$ takes the form:

$$
\begin{aligned}
\mathcal{Z} & =\frac{1}{N} \int e^{-\beta \widetilde{W}} \mathcal{D}\left(\sigma_{i}^{\mathrm{b}}, \sigma_{i}^{\mathrm{c}}, u\right) \\
\widetilde{W} & \equiv \widetilde{E}-i E_{\text {ext }}, \quad \widetilde{E} \equiv E_{\text {el }}+\widetilde{E}_{\text {core }}
\end{aligned}
$$

where $E_{\text {ext }}$ is given by (4), and $\widetilde{E}_{\text {core }}$ is expressed as follows:

$$
\widetilde{E}_{\text {core }} \equiv \frac{-1}{2 \ell} \int \sigma_{i}^{\mathrm{c}} \Delta^{-1} \sigma_{i}^{\mathrm{c}} \mathrm{d}^{2} x
$$

The functional $\widetilde{W}$ includes the elastic energy of non-singular dislocations $E_{\mathrm{el}}$, while the core energies are given by (17). The representations expressed either by (11), (21), (41), or by (15), (16), (17) are equivalent, i.e., the same Eqs. (15) and (9) arise for $f^{\mathrm{b}}$ and $f^{\mathrm{c}}$, respectively.

We are mainly interested in the exponential that contributes to the approximate expression for $\mathcal{Z}(15): \mathcal{Z} \simeq(\operatorname{Det} A)^{-1} e^{-\beta \mathcal{W}}$, where $\mathrm{A}$ is a kernel of the integral operator related to the second variation of $\widetilde{W}[43$. To derive $\mathcal{W}$, it is necessary to substitute the solutions $f^{\mathrm{b}}, f^{\mathrm{c}}$ into $\widetilde{W}(16)$ and apply the Green theorem. We neglect the terms exponentially small at $\kappa R \gg 1$, as well as those contributions which are irrelevant provided the differences $\left|\mathbf{y}_{I J}\right| \equiv\left|\mathbf{y}_{I}-\mathbf{y}_{J}\right|$ are moderate in comparison with $R$. The "electro-neutrality" condition $\sum_{I=1}^{\mathcal{N}} b_{I}=0$ prevents divergency caused by a large $\log R$. Finally, the effective energy $\mathcal{W}$ takes the form in the case of $\mathcal{N}$ dislocations:

$$
\mathcal{W}=2 \mu \int \partial_{1} e_{2}^{P}(\mathbf{x})\left(\left(\Delta-\kappa^{2}\right)^{-1}-\Delta^{-1}\right) \partial_{1} e_{2}^{P}(\mathbf{s}) \mathrm{d}^{2} x \mathrm{~d}^{2} s
$$

where the plastic sources are expressed like in (5), i.e., $\partial_{1} e_{2}^{P}=-\rho / 2$. Transforming $\mathcal{W}$ (18) further, we represent it as the potential dependent on the dislocation positions $\left\{\mathbf{y}_{I}\right\} \equiv\left\{\mathbf{y}_{I}\right\}_{1 \leq I \leq \mathcal{N}}:$

$$
\begin{aligned}
\mathcal{W} & =\mathcal{W}\left(\left\{\mathbf{y}_{I}\right\}\right)= \\
& =\frac{-\mu}{4 \pi} \int \rho(\mathbf{x})\left[\log |\mathbf{x}-\mathbf{s}|+K_{0}(\kappa|\mathbf{x}-\mathbf{s}|)\right] \rho(\mathbf{s}) \mathrm{d}^{2} x \mathrm{~d}^{2} s \\
& =\frac{-\mu}{4 \pi}\left(\sum_{I \neq J} b_{I} b_{J}\left[\log \left|\mathbf{y}_{I}-\mathbf{y}_{J}\right|+K_{0}\left(\kappa\left|\mathbf{y}_{I}-\mathbf{y}_{J}\right|\right)\right]+\log \left(\frac{2}{\kappa \gamma}\right) \sum_{I} b_{I}^{2}\right), \\
& =\frac{-\mu}{4 \pi} \sum_{I \neq J} b_{I} b_{J} \mathcal{U}\left(\kappa\left|\mathbf{y}_{I}-\mathbf{y}_{J}\right|\right),
\end{aligned}
$$

where $\mathcal{U}(\cdot)$ is defined as

$$
\mathcal{U}(s) \equiv \log \left(\frac{\gamma}{2} s\right)+K_{0}(s)
$$

and $\log \left(\frac{2}{\kappa \gamma}\right)$ is the core energy of the dislocation in the Coulomb gas representation. The distances in (19) are referred, for simplicity, to unit lattice spacing of a cubic crystal.

We turn to calculation of the force $F_{I J}=-\frac{\mathrm{d} \mathcal{W}}{\mathrm{d}\left|\mathbf{y}_{I J}\right|}$. Its expression,

$$
\frac{F_{I J}}{L}=\frac{\mu b_{I} b_{J} \kappa}{2 \pi} f\left(\kappa\left|\mathbf{y}_{I J}\right|\right), \quad f(s) \equiv \frac{\mathrm{d} \mathcal{U}}{\mathrm{d} s}=s^{-1}-K_{1}(s),
$$


demonstrates that $\mathcal{W}(19)$ at large separation $\left|\mathbf{y}_{I J}\right|$ corresponds to the energy of the Coulomb attraction between two charges of unlike signs $\left(b_{I} b_{J}<0\right)$. The maximal attraction of two opposite dislocations occurs at $\left|\mathbf{y}_{I}-\mathbf{y}_{J}\right| \approx \frac{1.1}{\kappa}$.

Consider a thermodynamical ensemble of positive and negative modified screw dislocations located, respectively, at $\left\{\mathbf{y}_{I}^{+}\right\}_{1 \leq I \leq \mathcal{N}}$ and $\left\{\mathbf{y}_{I}^{-}\right\}_{1 \leq I \leq \mathcal{N}}$ and possessing unit Burgers vectors. By analogy with the case of the two-dimensional electro-neutral plasma of positive and negative charges [46], the corresponding grand-canonical partition function $\mathbf{Z}_{C}$ is written, with regard at (19), as follows:

$$
\begin{aligned}
\mathbf{Z}_{C} & =\sum_{\mathcal{N}=0}^{\infty} \frac{1}{\mathcal{N} ! \mathcal{N} !} \prod_{I=1}^{\mathcal{N}} \int \mathrm{d}^{2} \mathbf{y}_{I}^{+} \prod_{J=1}^{\mathcal{N}} \int \mathrm{d}^{2} \mathbf{y}_{J}^{-} \exp [-2 \beta \mathcal{N} \Lambda+ \\
& \left.+\frac{\beta \mu}{4 \pi}\left(\sum_{I \neq J} \mathcal{U}\left(\kappa\left|\mathbf{y}_{I}^{+}-\mathbf{y}_{J}^{+}\right|\right)+\sum_{I \neq J} \mathcal{U}\left(\kappa\left|\mathbf{y}_{I}^{-}-\mathbf{y}_{J}^{-}\right|\right)-2 \sum_{I \neq J} \mathcal{U}\left(\kappa\left|\mathbf{y}_{I}^{+}-\mathbf{y}_{J}^{-}\right|\right)\right)\right]
\end{aligned}
$$

Here, $2 \mathcal{N}$ is the number of dislocations, and $\Lambda$ is the chemical potential per dislocation. The representation (22) implies that the summation over possible positions of the dislocations is replaced by the integration. The potential $\mathcal{U}(s)(20)$ is present in (22) instead of the regularised logarithm used in [50,51] for the case of the world-sheet vortices. It is appropriate to recall that the Coulomb gas of point charges is related to the two-dimensional sine-Gordon field theory [63 66].

\section{The correlation functions}

\subsection{Field-theoretical derivation of the stress-stress correlation function}

Gaining the experience of the derivation of the partition function (22), we pass on to the investigation of the relevant correlation functions. Let us take the field theory presented in section 2 as a starting point. Define the two-point stress-stress correlation functions $\left\langle\sigma_{i}^{\#}\left(\mathbf{x}_{1}\right) \sigma_{j}^{\#}\left(\mathbf{x}_{2}\right)\right\rangle_{P}$ (\# is either b or c) by means of the following functional averages [40 45]:

$$
\left\langle\sigma_{i}^{\#}\left(\mathbf{x}_{1}\right) \sigma_{j}^{\#}\left(\mathbf{x}_{2}\right)\right\rangle_{P} \equiv \frac{1}{\mathcal{Z}_{o}} \int \sigma_{i}^{\#}\left(\mathbf{x}_{1}\right) \sigma_{j}^{\#}\left(\mathbf{x}_{2}\right) e^{-\beta W} \mathcal{D}\left(\sigma_{i}^{\mathrm{b}}, \sigma_{i}^{\mathrm{c}}, u, e_{i}\right),
$$

where the functional $W$ is expressed by (2) and (41), and the index $P$ points out that the correlators (23) are defined with respect to a prescribed dislocation distribution specified in terms of the plastic strain $e_{i}^{P}(i=1,2)$. The latter is accounted for through the dependence of the functional $W=W\left(\mathcal{P}_{i}\right)$ on the source $\mathcal{P}_{i}$. In turn, the normalization factor $\mathcal{Z}_{o}$ is given by (11) though with $W$ taken at $\mathcal{P}_{i}=0$ and $\ell=0$. In other words, $\mathcal{Z}_{o}$ is the partiton function of a defectless state.

Approach of the generating functional is a natural way to evaluate (23) in the framework of the functional integration. We introduce the generating functional $\mathcal{G}\left[\mathbf{J}^{\mathrm{b}}, \mathbf{J}^{\mathrm{c}} \mid \mathcal{P}\right]$ as the functional integral parametrized by two auxiliary sources $\mathbf{J}^{\mathrm{b}}$ and $\mathbf{J}^{\mathrm{c}}$, as well as by the source $\mathcal{P}$ :

$$
\mathcal{G}\left[\mathbf{J}^{\mathrm{b}}, \mathbf{J}^{\mathrm{c}} \mid \mathcal{P}\right]=\int e^{-\beta W+i \beta \int\left(J_{i}^{\mathrm{b}} \sigma_{i}^{\mathrm{b}}+J_{j}^{\mathrm{c}} \sigma_{j}^{\mathrm{c}}\right) \mathrm{d}^{2} x} \mathcal{D}\left(\sigma_{i}^{\mathrm{b}}, \sigma_{i}^{\mathrm{c}}, u, e_{i}\right)
$$


Here the normalization factor is included into the integration measure, and each boldfaced notation, $\mathbf{J}^{\#}$ (\# is b or c) or $\mathcal{P}$, implies two components, say, $J_{1}^{\#}$ and $J_{2}^{\#}$. The source $\mathcal{P}$ is standing separately in left-hand side of (24) since its meaning is different. The correlators we are interested in, $\left\langle\sigma_{i}^{\#}\left(\mathbf{x}_{1}\right) \sigma_{j}^{\#}\left(\mathbf{x}_{2}\right)\right\rangle_{P}$, arise as follows:

$$
\left\langle\sigma_{i}^{\#}\left(\mathbf{x}_{1}\right) \sigma_{j}^{\#}\left(\mathbf{x}_{2}\right)\right\rangle_{P}=\lim _{\mathbf{J}^{\mathrm{b}}, \mathbf{J}^{\mathrm{c}} \rightarrow 0}\left(\mathcal{G}^{-1}\left[\mathbf{J}^{\mathrm{b}}, \mathbf{J}^{\mathrm{c}} \mid \mathcal{P}_{o}^{\mathrm{ph}}\right] \frac{(-i / \beta)^{2} \delta^{2}}{\delta J_{i}^{\#}\left(\mathbf{x}_{1}\right) \delta J_{j}^{\#}\left(\mathbf{x}_{2}\right)} \mathcal{G}\left[\mathbf{J}^{\mathrm{b}}, \mathbf{J}^{\mathrm{c}} \mid \mathcal{P}^{\mathrm{ph}}\right]\right),
$$

where $\mathcal{P}^{\text {ph }}$ is substituted for $\mathcal{P}\left(=\mathbf{e}^{P}+\mathbf{C}\right)$ in order to express that $\mathcal{G}$ is taken for a definite parametrization of the background configuration in terms of the field $\mathbf{C}$ (the appendix provides the calculation of $\left.\mathcal{G}\left[\mathbf{J}^{\mathrm{b}}, \mathbf{J}^{\mathrm{c}} \mid \mathcal{P}^{\mathrm{ph}}\right]\right)$. Moreover, $\left.\mathcal{P}_{o}^{\mathrm{ph}} \equiv \mathcal{P}^{\mathrm{ph}}\right|_{\mathbf{e}^{P}=0}$, i.e., the normalization is taken with respect of a defectless state, as in (23).

We shall obtain the correlation function $\left\langle\sigma_{i}^{\text {tot }}\left(\mathbf{x}_{1}\right) \sigma_{j}^{\text {tot }}\left(\mathbf{x}_{2}\right)\right\rangle_{P}$ of the physical stress field $\sigma_{i}^{\text {tot }}(\mathbf{x}) \equiv \frac{1}{i}\left(\sigma_{i}^{\mathrm{b}}(\mathbf{x})+\sigma_{i}^{\mathrm{c}}(\mathbf{x})\right)$. First, we calculate the functional (24) by shifts of the integration variables which cancel the terms linear in $\mathbf{J}^{\mathrm{b}}, \mathbf{J}^{\mathrm{c}}$. It is appropriate to obtain the answer in terms of a single source $J_{i}(\mathbf{x})=J_{i}^{\mathrm{b}}(\mathbf{x})=J_{i}^{\mathrm{c}}(\mathbf{x})$. The result is of the form:

$$
\mathcal{G}\left[\mathbf{J}, \mathbf{J} \mid \mathcal{P}^{\mathrm{ph}}\right]=e^{-\mu \beta Q / 2} \mathcal{G}\left[0,0 \mid \mathcal{P}_{o}^{\mathrm{ph}}\right]
$$

where

$$
\begin{aligned}
Q & \equiv \int\left(\partial_{i} \mathcal{J}_{i}\left(\frac{1}{\Delta}-\frac{1}{\Delta-\kappa^{2}}\right) \partial_{k} \mathcal{J}_{k}-\mathcal{J}_{i} \frac{\kappa^{2}}{\Delta-\kappa^{2}} \mathcal{J}_{i}+\Delta J_{i} \frac{1}{\Delta-\kappa^{2}} J_{i}\right) \mathrm{d}^{2} x, \\
\mathcal{J}_{i} & \equiv J_{i}-2 e_{i}^{P}
\end{aligned}
$$

(the Green functions $\Delta^{-1},\left(\Delta-\kappa^{2}\right)^{-1}$ imply the convolution operators). Then the physical correlation functions arise as follows:

$$
\left\langle\sigma_{i}^{\text {tot }}\left(\mathbf{x}_{1}\right) \sigma_{j}^{\text {tot }}\left(\mathbf{x}_{2}\right)\right\rangle_{P}=\lim _{\mathbf{J} \rightarrow 0}\left(\mathcal{G}^{-1}\left[\mathbf{J}, \mathbf{J} \mid \mathcal{P}_{o}^{\mathrm{ph}}\right] \frac{\beta^{-2} \delta^{2}}{\delta J_{i}\left(\mathbf{x}_{1}\right) \delta J_{j}\left(\mathbf{x}_{2}\right)} \mathcal{G}\left[\mathbf{J}, \mathbf{J} \mid \mathcal{P}^{\mathrm{ph}}\right]\right) .
$$

We calculate (28) using (26) and (27), and obtain:

$$
\left\langle\sigma_{i}^{\mathrm{tot}}\left(\mathbf{x}_{1}\right) \sigma_{j}^{\mathrm{tot}}\left(\mathbf{x}_{2}\right)\right\rangle_{P}=\frac{\mu^{2}}{4} e^{-\beta \mathcal{W}} \lim _{\mathbf{J} \rightarrow 0}\left[\left(\delta_{J_{i}\left(\mathbf{x}_{1}\right)} Q\right)\left(\delta_{J_{j}\left(\mathbf{x}_{2}\right)} Q\right)-\frac{2}{\mu \beta} \delta_{J_{i}\left(\mathbf{x}_{1}\right)} \delta_{J_{j}\left(\mathbf{x}_{2}\right)} Q\right],
$$

where $\delta_{J_{i}(\mathbf{x})} \equiv \delta / \delta J_{i}(\mathbf{x})$, and $\mathcal{W}, Q$ are given by (19) and (27). Equation (29) is reexpressed after the variational differentiations:

$$
\left\langle\sigma_{i}^{\mathrm{tot}}\left(\mathbf{x}_{1}\right) \sigma_{j}^{\mathrm{tot}}\left(\mathbf{x}_{2}\right)\right\rangle_{P}=e^{-\beta \mathcal{W}}\left[\sigma_{i}^{\mathrm{tot}}\left(\mathbf{x}_{1}\right) \sigma_{j}^{\mathrm{tot}}\left(\mathbf{x}_{2}\right)-\frac{\mu}{2 \pi \beta} \partial_{\left(\mathbf{x}_{1}\right)_{i}} \partial_{\left(\mathbf{x}_{2}\right)_{j}} \mathcal{U}\left(\kappa\left|\mathbf{x}_{1}-\mathbf{x}_{2}\right|\right)\right],
$$

where

$$
\sigma_{i}^{\text {tot }}(\mathbf{x})=\frac{\mu}{\pi} \epsilon_{i k} \partial_{(\mathbf{x})_{k}} \int \mathcal{U}(\kappa|\mathbf{x}-\mathbf{s}|) \partial_{1} e_{2}^{P}(\mathbf{s}) \mathrm{d} \mathbf{s}
$$

is the total elastic stress of the dislocational configuration. The exponential factor in (30) is the Boltzmann weight containing in its exponent the energy of the array of the dislocations.

Consider, for a comparison, our theory without the influence of the core energy, i.e., at $\ell=0$. Derive, firstly, the stress-stress correlator $\left\langle\sigma_{i}^{\mathrm{b}}\left(\mathbf{x}_{1}\right) \sigma_{j}^{\mathrm{b}}\left(\mathbf{x}_{2}\right)\right\rangle$ in the absence of 
the defects. The source $e_{i}^{P}$ being treated as unphysical one (that becomes zero after the variation) enables us to obtain:

$$
\left\langle\sigma_{i}^{\mathrm{b}}\left(\mathbf{x}_{1}\right) \sigma_{j}^{\mathrm{b}}\left(\mathbf{x}_{2}\right)\right\rangle=\frac{-\mu}{2 \pi \beta} \partial_{\left(\mathbf{x}_{1}\right)_{i}} \partial_{\left(\mathbf{x}_{2}\right)_{j}} \log \left|\mathbf{x}_{1}-\mathbf{x}_{2}\right| .
$$

Let the plastic source be the physical one. Then we obtain:

$$
\begin{aligned}
& \left\langle\sigma_{i}^{\mathrm{b}}\left(\mathbf{x}_{1}\right) \sigma_{j}^{\mathrm{b}}\left(\mathbf{x}_{2}\right)\right\rangle_{P}=e^{-\beta \mathcal{W}}\left[\frac{-\mu}{2 \pi \beta} \partial_{\left(\mathbf{x}_{1}\right)_{i}} \partial_{\left(\mathbf{x}_{2}\right)_{j}} \log \left|\mathbf{x}_{1}-\mathbf{x}_{2}\right|\right. \\
& \left.+\frac{\mu^{2}}{4 \pi^{2}} \sum_{I, J} b_{I} b_{J}\left(\epsilon_{i k} \partial_{\left(\mathbf{x}_{1}\right)_{k}} \log \left|\mathbf{x}_{1}-\mathbf{y}_{I}\right|\right)\left(\epsilon_{j l} \partial_{\left(\mathbf{x}_{2}\right)_{l}} \log \left|\mathbf{x}_{2}-\mathbf{y}_{J}\right|\right)\right],
\end{aligned}
$$

where $\mathcal{W}$ is given by (19), although with $\mathcal{U}$ replaced appropriately by the logarithmic potential.

The representation (30) corresponds to a specific spatial distribution of collection of the dislocations. We shall average $\left\langle\sigma_{i}^{\text {tot }}\left(\mathbf{x}_{1}\right) \sigma_{j}^{\text {tot }}\left(\mathbf{x}_{2}\right)\right\rangle_{P}$ over positions of the defects.

\subsection{Mean square of the dipole momentum}

Let us investigate the grand-canonical ensemble of the dislocations using the so-called dipole phase approximation which corresponds to pairs of the dislocations with opposite signs (located in $\mathbf{x}_{I}^{ \pm}, 1 \leq I \leq \mathcal{N}$ ) bound into "molecules". The corresponding partition function is specialized as follows [50]:

$$
\begin{aligned}
\mathbf{Z}_{\text {dip }} & =\sum_{\mathcal{N}=0}^{\infty} \frac{1}{\mathcal{N} !} \prod_{I=1}^{\mathcal{N}} \int \mathrm{d}^{2} \boldsymbol{\xi}_{I} \int \mathrm{d}^{2} \boldsymbol{\eta}_{I} \exp [-2 \beta \mathcal{N} \Lambda- \\
& \left.-\beta\left(\sum_{I=1}^{\mathcal{N}} w\left(\boldsymbol{\eta}_{I}\right)+\sum_{I \neq J} w_{I J}\right)\right]
\end{aligned}
$$

where $w\left(\boldsymbol{\eta}_{I}\right)$ is the energy of $I^{\text {th }}$ dipole centered in $\boldsymbol{\xi}_{I}=\left(\mathbf{x}_{I}^{+}+\mathbf{x}_{I}^{-}\right) / 2$ with the dipole momentum $\boldsymbol{\eta}_{I}=\mathbf{x}_{I}^{+}-\mathbf{x}_{I}^{-}$, while $w_{I J}$ is the energy of interaction between $I^{\text {th }}$ and $J^{\text {th }}$ dipoles.

Firstly, we calculate the mean square of the dipole momentum for a single molecule:

$$
\left\langle\boldsymbol{\eta}^{2}\right\rangle=\frac{\int \exp (-\beta w(\boldsymbol{\eta})) \boldsymbol{\eta}^{2} \mathrm{~d} \boldsymbol{\eta}}{\int \exp (-\beta w(\boldsymbol{\eta})) \mathrm{d} \boldsymbol{\eta}} .
$$

The average (35) has been calculated in [4] for electrically-neutral gas of particles with charges $\pm q$ interacting through the potential:

$$
\begin{aligned}
2 \Lambda-2 q_{I} q_{J} \log \frac{\left|\mathbf{x}_{I}-\mathbf{x}_{J}\right|}{a}, & \left|\mathbf{x}_{I}-\mathbf{x}_{J}\right|>a \\
0, & \left|\mathbf{x}_{I}-\mathbf{x}_{J}\right|<a
\end{aligned}
$$

where $\mathbf{x}_{I}$ is the $I^{\text {th }}$ charge position, and $a$ is the cutoff (e.g., the particle diameter, or lattice spacing). Moreover, $2 \Lambda$ is the energy necessary to create a pair of opposite charges 
at the distance $a$. The dipole energy is given by $\beta w(\boldsymbol{\eta})=\mathcal{K} \log (|\boldsymbol{\eta}| / a)$, where $\mathcal{K}=2 \beta q^{2}$. The average (35) takes the form [4]:

$$
\left\langle\boldsymbol{\eta}^{2}\right\rangle=a^{2} \frac{\mathcal{K}-2}{\mathcal{K}-4}=a^{2} \frac{\beta q^{2}-1}{\beta q^{2}-2},
$$

provided that $\beta q^{2}>2$. Therefore, $\left\langle\boldsymbol{\eta}^{2}\right\rangle \approx a^{2}$ in the limit of zero temperature, while $\left\langle\boldsymbol{\eta}^{2}\right\rangle$ infinitely grows at the temperature $T$ near its critical value $T_{c}$ given by $\beta_{c} q^{2}=2$.

Two-particle potential studied in [50] is expressed as $\beta w(\boldsymbol{\eta})=2 \pi \mathcal{K U}(|\boldsymbol{\eta}|)$, where $\mathcal{K}=2 \beta q^{2}$ (here $\beta$ is an effective parameter viewed as inverse temperature), and $\mathcal{U}(\eta)$ is regularised at small $\eta \equiv|\boldsymbol{\eta}|, \mathcal{U}(\eta)=\frac{1}{2} \log \frac{a^{2}+\eta^{2}}{a^{2}}$. We obtain from (35) at $\mathcal{K}>2 / \pi$ :

$$
\left\langle\boldsymbol{\eta}^{2}\right\rangle=a^{2} \frac{1}{\pi \mathcal{K}-2} .
$$

The dipole momentum (37) is strictly smaller than $\left\langle\boldsymbol{\eta}^{2}\right\rangle(\underline{36})$ at $\mathcal{K}>4$, i.e., in the second case the molecules are more compact at small enough temperatures.

The present paper deals with the dipole energy $\mathcal{U}(20)$, and the average (35) is specified as follows:

$$
\kappa^{2}\left\langle\boldsymbol{\eta}^{2}\right\rangle=\frac{\int_{0}^{\infty} \exp \left(-\mathcal{K}\left(\log \eta+K_{0}(\eta)\right)\right) \eta^{3} \mathrm{~d} \eta}{\int_{0}^{\infty} \exp \left(-\mathcal{K}\left(\log \eta+K_{0}(\eta)\right)\right) \eta \mathrm{d} \eta},
$$

where $\mathcal{K}=\mu b^{2} \beta / 2 \pi$. The integral in the nominator of (38) diverges at $\mathcal{K}<4$. Since dissociation is most probable for pairs of the dislocations with unit Burgers vectors, the dipolar phase does not exist at the temperature $T>T_{c} \equiv \frac{\mu}{8 \pi}$.

It has been proposed in [31, 34, 36] to represent the radius of the dislocation core $r_{c}$ in the form $r_{c} \simeq \eta_{b} / \kappa$, where $\eta_{b}=4.0$ [31], $\eta_{b}=6.0$ [36], or $\eta_{b}=10.0$ [34]. In turn, $\kappa^{-1}$ has been selected in terms of interatomic spacing $a$ as follows: $\kappa^{-1}=0.25 a$ [31], $\kappa^{-1}=0.399 a$ [30, 36]. For instance, $r_{c} \approx a$ according to [31, while $r_{c} \approx 2.4 a$ according to [30,36]. We represent the ratio (38) at $\mathcal{K}$ close enough to its "critical" value as follows:

$$
\left\langle\boldsymbol{\eta}^{2}\right\rangle \simeq \frac{2 \eta_{b}^{2}}{\kappa^{2}\left(1+2 \eta_{b}^{2} \mathrm{~A}^{-1}\right)} \frac{1}{\mathcal{K}-4}, \quad \mathrm{~A}^{-1} \equiv \int_{0}^{\eta_{b}} \exp \left(-4\left(\log \eta+K_{0}(\eta)\right)\right) \eta \mathrm{d} \eta,
$$

where $\eta_{b}$ and $\kappa$ are taken according to [30], [31] or [36]. Moreover, double-sided estimates can be obtained for $\mathcal{U}(\eta)(20)$ by adjusting appropriate trial functions. Such estimates enable one to demonstrate that dependence of $\left\langle\boldsymbol{\eta}^{2}\right\rangle$ (38) on growing $\mathcal{K}$ is characterized by the double inequality:

$$
\frac{1}{\mathcal{K}^{2}}<\kappa^{2}\left\langle\boldsymbol{\eta}^{2}\right\rangle<\frac{1}{\mathcal{K}}
$$

The potential $\mathcal{U}(\eta)=\frac{1}{2} \log \left(1+\eta^{2}\right)$ can be used in (38) instead of $\mathcal{U}(20)$ for a comparison of extensions of the dipole momenta. According to the estimate (40), the dipoles are more compact for $\mathcal{U}(20)$ at small enough temperatures.

\subsection{The correlator of two stresses}

Now, we turn to the averaging of the correlation functions (30) over the dipole positions. We shall follow [50] where the asymptotic behaviour of the correlation functions of the 
string models have been investigated in the presence of the vortex dipoles. We shall denote the new average as $\left\langle\left\langle\sigma_{i}^{\text {tot }}\left(\mathbf{x}_{1}\right) \sigma_{j}^{\text {tot }}\left(\mathbf{x}_{2}\right)\right\rangle\right\rangle$, and its expression arises as follows:

$$
\begin{aligned}
\left\langle\left\langle\sigma_{i}^{\text {tot }}\left(\mathbf{x}_{1}\right) \sigma_{j}^{\text {tot }}\left(\mathbf{x}_{2}\right)\right\rangle\right\rangle & =\frac{-\mu}{2 \pi \beta} \partial_{\left(\mathbf{x}_{1}\right)_{i}} \partial_{\left(\mathbf{x}_{2}\right)_{j}} \mathcal{U}\left(\kappa\left|\mathbf{x}_{1}-\mathbf{x}_{2}\right|\right) \\
& +\mathbf{Z}_{\text {dip }}^{-1} \sum_{\substack{\text { numbers of dipoles, } \\
\text { dipole positions }}} \sigma_{i}^{\text {tot }}\left(\mathbf{x}_{1}\right) \sigma_{j}^{\text {tot }}\left(\mathbf{x}_{2}\right) e^{-\beta \mathcal{W}}
\end{aligned}
$$

where $\mathbf{Z}_{\text {dip }}$ is the partition function (34). The dipole positions are confined within a disc of radius $R$.

According to (40), the dipoles are very compact since the dipole momenta are not too large at small enough temperature: $\left\langle\boldsymbol{\eta}^{2}\right\rangle \ll \kappa^{-2}$. Therefore, summation over the dipole positions can be replaced by integration. We use the dipole's center of mass and momentum coordinates, respectively, $\boldsymbol{\xi}_{L}=\left(\mathbf{y}_{L}^{+}+\mathbf{y}_{L}^{-}\right) / 2$ and $\boldsymbol{\eta}_{L}=\mathbf{y}_{L}^{+}-\mathbf{y}_{L}^{-}$for each pair of opposite dislocations at $\mathbf{y}_{L}^{ \pm}$forming the $L^{\text {th }}$ dipole $(1 \leq L \leq \mathcal{N})$. We continue to use the approximation of non-interacting dipoles, and the sum in right-hand side of (41) takes the form:

$$
\begin{aligned}
& \sum_{\text {numbers }, \text { positions }} \sigma_{i}^{\text {tot }}\left(\mathbf{x}_{1}\right) \sigma_{j}^{\text {tot }}\left(\mathbf{x}_{2}\right) e^{-\beta \mathcal{W}}= \\
= & \left(\frac{\mu b}{2 \pi}\right)^{2} \epsilon_{i k} \epsilon_{j l} \partial_{\left(\mathbf{x}_{1}\right)_{k}} \partial_{\left(\mathbf{x}_{2}\right)_{l}} \sum_{\mathcal{N}=1}^{\infty} \frac{1}{\mathcal{N} !} \prod_{I=1}^{\mathcal{N}} \int \mathrm{d}^{2} \boldsymbol{\xi}_{I} \int \mathrm{d}^{2} \boldsymbol{\eta}_{I} e^{-\beta\left(2 \Lambda+w\left(\boldsymbol{\eta}_{I}\right)\right)} \\
\times & \sum_{K, L=1}^{\mathcal{N}}\left(\mathcal{U}\left(\kappa\left|\mathbf{x}_{1}-\mathbf{y}_{K}^{+}\right|\right)-\mathcal{U}\left(\kappa\left|\mathbf{x}_{1}-\mathbf{y}_{K}^{-}\right|\right)\right)\left(\mathcal{U}\left(\kappa\left|\mathbf{x}_{2}-\mathbf{y}_{L}^{+}\right|\right)-\mathcal{U}\left(\kappa\left|\mathbf{x}_{2}-\mathbf{y}_{L}^{-}\right|\right)\right),
\end{aligned}
$$

where $\mathcal{N}$ is the number of dipoles, and we use for $\sigma_{i}^{\text {tot }}(\mathbf{x})$ the representation (31). We estimate $\left|\boldsymbol{\eta}_{L}\right| \ll\left|\mathbf{x}-\boldsymbol{\xi}_{L}\right|$ (compactness of the dipoles) and adopt in leading approximation [50]:

$$
\mathcal{U}\left(\kappa\left|\mathbf{x}-\mathbf{y}_{L}^{+}\right|\right)-\mathcal{U}\left(\kappa\left|\mathbf{x}-\mathbf{y}_{L}^{-}\right|\right) \approx-\left(\boldsymbol{\eta}_{L}, \partial_{\mathbf{x}}\right) \mathcal{U}\left(\kappa\left|\mathbf{x}-\boldsymbol{\xi}_{L}\right|\right),
$$

where $(\cdot, \cdot)$ stands for the scalar product of 2 -vectors. The relation (43) allows us to re-express (42) as follows:

$$
\begin{aligned}
& \left(\frac{\mu b}{2 \pi}\right)^{2} \epsilon_{i k} \epsilon_{j l} \partial_{\left(\mathbf{x}_{1}\right)_{k}} \partial_{\left(\mathbf{x}_{2}\right)_{l}} \sum_{\mathcal{N}=1}^{\infty} \frac{1}{\mathcal{N} !} \prod_{I=1}^{\mathcal{N}} \int \mathrm{d}^{2} \boldsymbol{\xi}_{I} \int \mathrm{d}^{2} \boldsymbol{\eta}_{I} e^{-\beta\left(2 \Lambda+w\left(\boldsymbol{\eta}_{I}\right)\right)} \\
& \times \sum_{L=1}^{\mathcal{N}}\left(\boldsymbol{\eta}_{L}, \partial_{\mathbf{x}_{1}}\right) \mathcal{U}\left(\kappa\left|\mathbf{x}_{1}-\boldsymbol{\xi}_{L}\right|\right)\left(\boldsymbol{\eta}_{L}, \partial_{\mathbf{x}_{2}}\right) \mathcal{U}\left(\kappa\left|\mathbf{x}_{2}-\boldsymbol{\xi}_{L}\right|\right) .
\end{aligned}
$$

To proceed with (44), essential technical task is to calculate the integral:

$$
\int \mathrm{d}^{2} \boldsymbol{\xi} \int \mathrm{d}^{2} \boldsymbol{\eta}\left(\boldsymbol{\eta}, \partial_{\mathbf{x}_{1}}\right) \mathcal{U}\left(\kappa\left|\mathbf{x}_{1}-\boldsymbol{\xi}\right|\right)\left(\boldsymbol{\eta}, \partial_{\mathbf{x}_{2}}\right) \mathcal{U}\left(\kappa\left|\mathbf{x}_{2}-\boldsymbol{\xi}\right|\right) e^{-\beta(2 \Lambda+w(\boldsymbol{\eta}))}
$$

First, we express the $\boldsymbol{\eta}$-integration by means of the relation

$$
\frac{\int \exp \left(-2 \beta \Lambda-\mathcal{K}\left(\log \left(\frac{\gamma}{2} \kappa \eta\right)+K_{0}(\kappa \eta)\right)\right) \eta_{i} \eta_{j} \mathrm{~d}^{2} \boldsymbol{\eta}}{\int \exp \left(-2 \beta \Lambda-\mathcal{K}\left(\log \left(\frac{\gamma}{2} \kappa \eta\right)+K_{0}(\kappa \eta)\right)\right) \mathrm{d}^{2} \boldsymbol{\eta}}=\frac{\delta_{i j}}{2}\left\langle\boldsymbol{\eta}^{2}\right\rangle,
$$


where $\mathcal{K}=\frac{\mu b^{2} \beta}{2 \pi}, \eta=|\boldsymbol{\eta}|$, and $\left\langle\boldsymbol{\eta}^{2}\right\rangle$ is given by (38). Then, after introducing the notation $\bar{N}$ for the dipole density [4, 50 ]:

$$
\bar{N} \equiv \int \exp \left(-2 \beta \Lambda-\mathcal{K}\left(\log \left(\frac{\gamma}{2} \kappa \eta\right)+K_{0}(\kappa \eta)\right)\right) \mathrm{d}^{2} \boldsymbol{\eta},
$$

the relation (46) allows us to re-express the integral (45) as follows:

$$
\frac{\left\langle\boldsymbol{\eta}^{2}\right\rangle \bar{N}}{2} \int\left(\partial_{\mathbf{x}_{1}} \mathcal{U}\left(\kappa\left|\mathbf{x}_{1}-\boldsymbol{\xi}\right|\right), \partial_{\mathbf{x}_{2}} \mathcal{U}\left(\kappa\left|\mathbf{x}_{2}-\boldsymbol{\xi}\right|\right)\right) \mathrm{d}^{2} \boldsymbol{\xi}
$$

The Green theorem enables one to carry out the $\boldsymbol{\xi}$-integration in (48). Eventually, we use the partition function $\mathbf{Z}_{\text {dip }}($ (34) ) and obtain:

$$
\begin{aligned}
& \left\langle\left\langle\sigma_{i}^{\text {tot }}\left(\mathbf{x}_{1}\right) \sigma_{j}^{\text {tot }}\left(\mathbf{x}_{2}\right)\right\rangle\right\rangle=\frac{-\mu}{2 \pi \beta} \partial_{\left(\mathbf{x}_{1}\right)_{i}} \partial_{\left(\mathbf{x}_{2}\right)_{j}} \mathcal{U}(\kappa|\Delta \mathbf{x}|) \\
& -\left\langle\boldsymbol{\eta}^{2}\right\rangle \bar{N} \frac{\mu^{2} b^{2}}{4 \pi} \epsilon_{i k} \epsilon_{j l} \partial_{\left(\mathbf{x}_{1}\right)_{k}} \partial_{\left(\mathbf{x}_{2}\right)_{l}}\left(\mathcal{U}(\kappa|\Delta \mathbf{x}|)+\frac{\kappa|\Delta \mathbf{x}|}{2} K_{1}(\kappa|\Delta \mathbf{x}|)\right),
\end{aligned}
$$

where (and henceforth) $\Delta \mathbf{x} \equiv \mathbf{x}_{1}-\mathbf{x}_{2}$.

At large separation of the arguments, $|\Delta \mathbf{x}| \gg \kappa^{-1}$, the asymptotic of (49) is governed by the logarithmic contribution since the modified Bessel functions decay exponentially. We use the rule of differentiation of the logarithm,

$$
\begin{aligned}
& \partial_{\left(\mathbf{x}_{1}\right)_{k}} \partial_{\left(\mathbf{x}_{2}\right)_{l}} \log \left|\mathbf{x}_{1}-\mathbf{x}_{2}\right|= \\
& =\frac{1}{\left|\mathbf{x}_{1}-\mathbf{x}_{2}\right|^{2}}\left(-\delta_{k l}+2 \frac{\left(\mathbf{x}_{1}-\mathbf{x}_{2}\right)_{k}\left(\mathbf{x}_{1}-\mathbf{x}_{2}\right)_{l}}{\left|\mathbf{x}_{1}-\mathbf{x}_{2}\right|^{2}}\right)-\pi \delta_{k l} \stackrel{(2)}{\delta}\left(\mathbf{x}_{1}-\mathbf{x}_{2}\right),
\end{aligned}
$$

as well as the relation $\epsilon_{i k} \epsilon_{j l}=\delta_{i j} \delta_{k l}-\delta_{i l} \delta_{k j}$, and obtain:

$$
\left\langle\left\langle\sigma_{i}^{\text {tot }}\left(\mathbf{x}_{1}\right) \sigma_{j}^{\text {tot }}\left(\mathbf{x}_{2}\right)\right\rangle\right\rangle=\left(\frac{-\mu}{2 \pi \beta}+\bar{N}\left\langle\boldsymbol{\eta}^{2}\right\rangle \frac{\mu^{2} b^{2}}{4 \pi}\right) \frac{2(\Delta \mathbf{x})_{i}(\Delta \mathbf{x})_{j}-\delta_{i j}|\Delta \mathbf{x}|^{2}}{|\Delta \mathbf{x}|^{4}}+o\left(|\Delta \mathbf{x}|^{-2}\right) .
$$

Therefore, the stress-stress correlator (being considered with dislocations as well as without them) decreases as $|\Delta \mathbf{x}|^{-2}$ at growing separation $|\Delta \mathbf{x}|$.

The asymptotic law $|\Delta \mathbf{x}|^{-2}$ is due to the analytical structure of the logarithm differentiated. This law being extrapolated to small distances, $|\Delta \mathbf{x}| \ll 1$, would imply a singular behaviour of the stress-stress correlator in question. In the present approach, the short-distance behaviour of the correlation function is less singular because of the core influence. Since the logarithmic contributions are canceled in the potential $\mathcal{U}$, the short-distance behaviour is also less singular:

$$
\begin{aligned}
\left\langle\left\langle\sigma_{i}^{\text {tot }}\left(\mathbf{x}_{1}\right) \sigma_{j}^{\text {tot }}\left(\mathbf{x}_{2}\right)\right\rangle\right\rangle & =\frac{-\mu}{2 \pi \beta} \frac{\kappa^{2}}{2}\left[-\delta_{i j}\left(\frac{1}{2}+\bar{N}\left\langle\boldsymbol{\eta}^{2}\right\rangle \frac{\mu b^{2} \beta}{4}-\log \left(\frac{\gamma}{2} \kappa|\Delta \mathbf{x}|\right)\right)\right. \\
& \left.+\frac{(\Delta \mathbf{x})_{i}(\Delta \mathbf{x})_{j}}{|\Delta \mathbf{x}|^{2}}+\mathcal{O}\left(|\Delta \mathbf{x}|^{2} \log |\Delta \mathbf{x}|\right)\right]
\end{aligned}
$$

We considered the approximation of non-interacting dipoles. Dipole-dipole interaction can also be taken into account in the present framework. Moreover, second order corrections to the stress fields inside the core could become important for sufficiently dense gas of the dipoles [39]. However, this should be a subject of a separate investigation. 


\section{The renormalization of the shear modulus}

The present section is to investigate the renormalization of the shear modulus caused by the modified screw dislocations. One should refer to the original paper [9] for the definition of the inverse of the tensor of renormalized elastic constants in the presence of dislocations. Further details on the dislocation contribution to the elastic constants can be found in [9] and [16] (devoted, respectively, to two- and three-dimensional situations). Adopting the relevant definitions [9, 16], we shall consider the following expression for the renormalized shear modulus $\mu_{\text {ren }}$ :

$$
\frac{1}{\mu_{\mathrm{ren}}} \equiv \frac{\beta}{\mu^{2} \mathcal{S}} \sum_{i, k=1,2} \iint\left\langle\left\langle\sigma_{i}^{\mathrm{tot}}\left(\mathbf{x}_{1}\right) \sigma_{k}^{\mathrm{tot}}\left(\mathbf{x}_{2}\right)\right\rangle\right\rangle \mathrm{d}^{2} \mathbf{x}_{1} \mathrm{~d}^{2} \mathbf{x}_{2},
$$

where the correlator is defined by (41), $\mathcal{S}$ is the area of the sample's cross-section. We use (49) and (50), and obtain:

$$
\begin{aligned}
& \sum_{k=1,2}\left\langle\left\langle\sigma_{k}^{\mathrm{tot}}\left(\mathbf{x}_{1}\right) \sigma_{k}^{\mathrm{tot}}\left(\mathbf{x}_{2}\right)\right\rangle\right\rangle=\frac{\mu \kappa^{2}}{2 \pi \beta}\left(K_{0}(\kappa|\Delta \mathbf{x}|)+\right. \\
& \left.+\frac{\alpha \mu}{2} \kappa|\Delta \mathbf{x}| K_{1}(\kappa|\Delta \mathbf{x}|)\right), \quad \alpha \equiv \frac{\beta b^{2}\left\langle\boldsymbol{\eta}^{2}\right\rangle \bar{N}}{2} .
\end{aligned}
$$

"Non-diagonal" correlators $\left\langle\left\langle\sigma_{k}^{\text {tot }}\left(\mathbf{x}_{1}\right) \sigma_{l}^{\text {tot }}\left(\mathbf{x}_{2}\right)\right\rangle\right\rangle, k \neq l$, are negligible with respect to the two integrations in (53). The parameter $\alpha$ (54) is proportional to the mean area covered by the dipoles, $2 \pi\left\langle\boldsymbol{\eta}^{2}\right\rangle \bar{N}$. Therefore, one obtains from (53) and (54) the following answer:

$$
\frac{1}{\mu_{\mathrm{ren}}}=\frac{1}{\mu} \mathcal{C}_{1}(\kappa R)+\alpha \mathcal{C}_{2}(\kappa R)
$$

where the functions $\mathcal{C}_{1}(\kappa R)$ and $\mathcal{C}_{2}(\kappa R)$ are given by the modified Bessel functions:

$$
\begin{aligned}
& \mathcal{C}_{1}(\kappa R)=1-2 K_{1}(\kappa R) I_{1}(\kappa R), \\
& \mathcal{C}_{2}(\kappa R)=2-2 I_{1}(\kappa R)\left(K_{1}(\kappa R)-\kappa R K_{1}^{\prime}(\kappa R)\right),
\end{aligned}
$$

and $K_{1}^{\prime}(z)=\frac{\mathrm{d}}{\mathrm{d} z} K_{1}(z)$. The renormalization rule (55) demonstrates the dependence of the shear modulus $\mu_{\text {ren }}$ on the dimensionless parameter $\kappa R$. The coefficients $\mathcal{C}_{1}(\kappa R)$ and $\mathcal{C}_{2}(\kappa R)$ both are positive and less than unity though tend to unity at $\kappa R \rightarrow \infty$. We obtain the estimates for $\mathcal{C}_{1}(\kappa R), \mathcal{C}_{2}(\kappa R)$ at increasing $\kappa R$ :

$$
\mathcal{C}_{1}(\kappa R) \approx 1-\frac{1}{\kappa R}+\ldots, \quad \mathcal{C}_{2}(\kappa R) \approx 1-\frac{3}{2 \kappa R}+\ldots
$$

where the ellipsis imply the terms $\mathcal{O}\left((\kappa R)^{-2}\right)$.

On the other hand, an analogue of (54) valid for singular screw dislocations takes the form:

$$
\sum_{k=1,2}\left\langle\left\langle\sigma_{k}^{\mathrm{b}}\left(\mathbf{x}_{1}\right) \sigma_{k}^{\mathrm{b}}\left(\mathbf{x}_{2}\right)\right\rangle\right\rangle=\frac{\mu}{\beta}(1+\alpha \mu) \stackrel{(2)}{\delta}\left(\mathbf{x}_{1}-\mathbf{x}_{2}\right) .
$$

The right-hand sides of (154) and (58) are weakly coinciding at $\kappa^{-1} \rightarrow 0$ (i.e., when the core's scale is shrunk). This can be demonstrated by means of integration with an appropriate trial function. Inserting (58) into (53) one obtains:

$$
\frac{1}{\mu_{\mathrm{ren}}}=\frac{1}{\mu}+\alpha
$$


The renormalization rule (59) is in agreement with that obtained in the original paper [4] with the help of the macroscopic stress function of collection of the dislocation dipoles. Equation (59) agrees with the renormalization of the shear modulus found in [9, 16] provided the concentration of the defects is low. As is seen from (59), increasing of $\alpha$ results in decreasing of $\mu_{\text {ren }}$. Clearly, Eq. (55) is reduced to (59) when $\kappa R$ tends to infinity, and so the unit values of $\mathcal{C}_{1}$ and $\mathcal{C}_{2}$ correspond to the case of singular dislocations. Roughly speaking, shrinking up the core regions one goes back to singular dislocations.

Equation (55) can be re-expressed as follows:

$$
\mu \rightarrow \mu_{\text {ren }}=\frac{\mu}{\mathcal{C}_{1}(\kappa R)}\left(1+\mu \alpha \frac{\mathcal{C}_{2}(\kappa R)}{\mathcal{C}_{1}(\kappa R)}\right)^{-1} .
$$

At finite $\kappa R$ (the scale $1 / \kappa$ is comparable with the sample's scale $R$ ), the non-triviality of the dislocation cores is valuable for the renormalization of the shear modulus. According to (57) and (60), the character of the decreasing of $\mu_{\text {ren }}$ is changed in comparison with the case of the singular dislocations: it is slower since $\mathcal{C}_{2}(\kappa R) / \mathcal{C}_{1}(\kappa R)<1$ at large $\kappa R$. Recall that the critical exponents of appropriate correlation functions are analogously renormalized because of the presence of the vortex pairs, for instance, in the two-dimensional Bose gas [6], in the classical planar Heisenberg model [47], or on the string world-sheets [50,51].

\section{Discussion}

An array of parallel singular screw dislocations is equivalent (as a planar system with respect of the sample's cross-section) to the two-dimensional Coulomb gas of charged point particles. The latter is a subject covered by the theory [1-14 of the phase transitions in the low-dimensional systems of condensed matter physics. Dislocations influence renormalization of the elastic constants of the corresponding material sample. This paper investigates the re-normalization of the shear modulus in the case of the screw dislocations possessing the core regions of finite-size. The transformation to the Coulomb-like system is used.

The approach of [35, 39] to description of singularityless dislocations is elaborated in the given paper further for studying the collection of the modified screw dislocations as a thermodynamic ensemble. Specifically, a long enough cylinder pierced by non-singular screw dislocations is studied. A field-theoretical formalism is developed for investigating the corresponding partition function in the form of the functional integral. The stress potentials of the modified dislocations play the role of its saddle points because of the choice of the energy functional. The plastic external source is involved which governs the background stress distribution. This is distinct from [34 37] where the usage of the so-called "null-Lagrangian" is responsible for arising of the background stress field.

Calculation of the partition function relates the system of dislocation dipoles to equivalent description of the electrically neutral Coulomb gas of charges interacting via potential which is logarithmic at large separation but tends to zero for the charges sufficiently close to each other. The smoothing of the Coulomb potential at short mutual separations occurs since the self-energy of the cores is accounted for. The stress-stress correlation functions are obtained and used for studying the renormalization of the shear modulus in the approximation of the dilute gas of the dislocation dipoles.

It is demonstrated that the renormalized shear modulus depends non-conventionally on the ratio $R / \kappa^{-1}=\kappa R$ of two lengths characterizing the sample's cross-section and the 
dislocation core sizes. Note that applicability of the effects of renormalization of the elastic constants to experimental observations is discussed in [16] for the case of singular defects. Since the contributions caused by the core are sensible at moderate $\kappa R$, it is hopeful that the formalism developed could be efficient for the nanotubes with comparable $R$ and $\kappa^{-1}$. With regard to [32,35, 37], it is hopeful that the formalism presented can be extended to a hollow cylinder, as well as developed further to cover the modified edge dislocations. The latter could be interesting as far as the physics of multi-layer nanotubes and wrapped crystals is concerned [22,25].

\section{Acknowledgement}

It is a pleasure to express my gratitude to N. M. Bogoliubov, M. Yu. Gutkin, M. O. Katanaev, and A. G. Pronko. The research described has been supported in part by RFBR (No. 10-01-00600) and by the Russian Academy of Sciences program "Mathematical Methods in Non-Linear Dynamics".

\section{Appendix}

Consider the generating functional $\mathcal{G}\left[\mathbf{J}^{\mathrm{b}}, \mathbf{J}^{\mathrm{c}} \mid \mathcal{P}\right](24)$ dependent on three 2-component sources, $\mathbf{J}^{\mathrm{b}}, \mathbf{J}^{\mathrm{c}}$, and $\mathcal{P}$ :

$$
\mathcal{G}\left[\mathbf{J}^{\mathrm{b}}, \mathbf{J}^{\mathrm{c}} \mid \mathcal{P}\right]=\int e^{-\beta W+i \beta \int\left(J_{i}^{\mathrm{b}} \sigma_{i}^{\mathrm{b}}+J_{j}^{\mathrm{c}} \sigma_{j}^{\mathrm{c}}\right) \mathrm{d}^{2} x} \mathcal{D}\left(\sigma_{i}^{\mathrm{b}}, \sigma_{i}^{\mathrm{c}}, u, e_{i}\right)
$$

The exponent in (A1) is specified, after fixing the "Coulomb gauge", as follows:

$$
\begin{aligned}
& -\beta W+i \beta \int\left(J_{i}^{\mathrm{b}} \sigma_{i}^{\mathrm{b}}+J_{j}^{\mathrm{c}} \sigma_{j}^{\mathrm{c}}\right) \mathrm{d}^{2} x= \\
& =\frac{-\beta}{2 \mu} \int\left(\sigma_{i}^{\mathrm{b}}+\sigma_{i}^{\mathrm{c}}\right)^{2} \mathrm{~d}^{2} x-2 \beta \ell \int e_{i} \Delta e_{i} \mathrm{~d}^{2} x \\
& +i \beta \int \sigma_{i}^{\mathrm{b}}\left(\partial_{i} u-2 \mathcal{P}_{i}+J_{i}^{\mathrm{b}}\right) \mathrm{d}^{2} x+\beta \int \sigma_{i}^{\mathrm{c}}\left(2 e_{i}+i J_{i}^{\mathrm{c}}\right) \mathrm{d}^{2} x .
\end{aligned}
$$

It is appropriate to calculate (A1) by shifts of the functional integration variables [40 45]. As a first step, the strain field $e_{i}$ should be integrated out by the shift

$$
e_{i} \longrightarrow e_{i}+\frac{1}{2 \ell \Delta} \sigma_{i}^{\mathrm{c}}
$$

After re-arrangements we obtain for $\mathcal{G}\left[\mathbf{J}^{\mathrm{b}}, \mathbf{J}^{\mathrm{c}} \mid \mathcal{P}\right]$ :

$$
\begin{aligned}
& \mathcal{G}\left[\mathbf{J}^{\mathrm{b}}, \mathbf{J}^{\mathrm{c}} \mid \mathcal{P}\right]=\text { const } \times \int \exp \left[\frac{-\beta}{2 \mu} \int \sigma_{i}^{\mathrm{c}} \mathrm{D}^{-1} \sigma_{i}^{\mathrm{c}} \mathrm{d}^{2} x+i \beta \int \sigma_{i}^{\mathrm{c}} J_{i}^{\mathrm{c}} \mathrm{d}^{2} x\right. \\
& \left.-\frac{\beta}{2 \mu} \int\left(\sigma_{i}^{\mathrm{b}} \sigma_{i}^{\mathrm{b}}-i 2 \mu \sigma_{i}^{\mathrm{b}}\left(\partial_{i} u-2 \mathcal{P}_{i}+J_{i}^{\mathrm{b}}+\frac{i}{\mu} \sigma_{i}^{\mathrm{c}}\right)\right) \mathrm{d}^{2} x\right] \mathcal{D}\left(\sigma_{i}^{\mathrm{b}}, \sigma_{i}^{\mathrm{c}}, u\right) .
\end{aligned}
$$

Decoupled integrations, being constant factors which are not of interest now, are systematically included into the prefactor const. The kernel $\mathrm{D}^{-1}$ is defined in (A3) as follows:

$$
\mathrm{D}^{-1} \equiv \delta-\frac{\kappa^{2}}{\Delta}
$$


where $\Delta^{-1}$ is the Green function of two-dimensional Laplacian, and $\delta$ is the delta-function.

Next step is to shift subsequently the variables $\sigma_{i}^{\mathrm{b}}$ and $\sigma_{i}^{\mathrm{c}}$. It is assumed that $J_{i}^{\mathrm{b}}=$ $J_{i}^{\mathrm{c}}=J_{i}$. After the shifts

$$
\begin{aligned}
& \sigma_{i}^{\mathrm{b}} \longrightarrow \sigma_{i}^{\mathrm{b}}+i \mu\left(\partial_{i} u-2 e_{i}^{P}+J_{i}\right), \\
& \sigma_{i}^{\mathrm{c}} \longrightarrow \sigma_{i}^{\mathrm{c}}-i \mu \mathrm{D}\left(\partial_{i} u-2 e_{i}^{P}\right),
\end{aligned}
$$

the generating functional takes the form:

$$
\begin{aligned}
& \mathcal{G}[\mathbf{J}, \mathbf{J} \mid \mathcal{P}]=\text { const } \times \int \exp \left[\frac { - \mu \beta } { 2 } \int \left(\left(\partial_{i} u+\mathcal{J}_{i}\right)(\delta+\mathrm{D})\left(\partial_{i} u+\mathcal{J}_{i}\right)+J_{i} \mathrm{D} J_{i}\right.\right. \\
& \left.-2\left(\partial_{i} u+\mathcal{J}_{i}\right)\left(\mathrm{D} J_{i}+2 C_{i}\right)\right) \mathrm{d}^{2} x-i \beta \int \sigma_{i}^{\mathrm{b}}\left(2 C_{i}-\mathrm{D}\left(\partial_{i} u-2 e_{i}^{P}\right)\right) \mathrm{d}^{2} x \\
& \left.-\frac{\beta}{2 \mu} \int\left(\sigma_{i}^{\mathrm{c}} \mathrm{D}^{-1} \sigma_{i}^{\mathrm{c}}+\sigma_{i}^{\mathrm{b}} \sigma_{i}^{\mathrm{b}}+2 \sigma_{i}^{\mathrm{b}} \sigma_{i}^{\mathrm{c}}\right) \mathrm{d}^{2} x\right] \mathcal{D}\left(\sigma_{i}^{\mathrm{b}}, \sigma_{i}^{\mathrm{c}}, u\right),
\end{aligned}
$$

where the operator $\mathrm{D}$ is inverse to $\mathrm{D}^{-1}(\mathrm{~A} 4)$, and $\mathcal{J}_{i} \equiv J_{i}-2 e_{i}^{P}$.

The experience of section 2 tells us about a necessity of fixing of the background contribution by means of a self-consistent choice of the functions $C_{i}$. From a viewpoint of the functional integration, the corresponding substitute

$$
C_{i}=\frac{1}{2} \mathrm{D}\left(\partial_{i} u-2 e_{i}^{P}\right)
$$

(which solves (10)) allows one to decouple the integration over $u$. The corresponding functional integral is just responsible for the dependence of the partition function on the plastic strain $e_{i}^{P}$ and thus on the defect distribution. Then the following representation arises:

$$
\mathcal{G}\left[\mathbf{J}, \mathbf{J} \mid \mathcal{P}^{\mathrm{ph}}\right]=\text { const } \times \int \exp \left[\frac{-\mu \beta}{2} \int\left(\left(\partial_{i} u+\mathcal{J}_{i}\right)(\delta-\mathrm{D})\left(\partial_{i} u+\mathcal{J}_{i}\right)+J_{i} \mathrm{D} J_{i}\right) \mathrm{d}^{2} x\right] \mathcal{D}(u),
$$

where the notation $\mathcal{P}^{\mathrm{ph}}$ implies that the arbitrariness due to $C_{i}$ is removed. As a final step, the shift

$$
u \longrightarrow u-\frac{1}{\Delta} \partial_{i} \mathcal{J}_{i}
$$

allows one to get rid of the contribution of the first order in $\partial_{i} u$. Finally, the generating functional takes the form:

$$
\begin{aligned}
\mathcal{G}\left[\mathbf{J}, \mathbf{J} \mid \mathcal{P}^{\mathrm{ph}}\right] & =\mathcal{G}\left[0,0 \mid \mathcal{P}_{o}^{\mathrm{ph}}\right] \times \exp \left[\frac { - \mu \beta } { 2 } \int \left(\partial_{i} \mathcal{J}_{i}\left(\frac{1}{\Delta}-\frac{1}{\Delta-\kappa^{2}}\right) \partial_{k} \mathcal{J}_{k}\right.\right. \\
& \left.\left.-\mathcal{J}_{i} \frac{\kappa^{2}}{\Delta-\kappa^{2}} \mathcal{J}_{i}+\Delta J_{i} \frac{1}{\Delta-\kappa^{2}} J_{i}\right) d^{2} x\right]
\end{aligned}
$$

where $\mathcal{J}_{i} \equiv J_{i}-2 e_{i}^{P}$. All the integrations decoupled are gathered within $\mathcal{G}\left[0,0 \mid \mathcal{P}_{o}^{\mathrm{ph}}\right]$, where $\left.\mathcal{P}_{o}^{\mathrm{ph}} \equiv \mathcal{P}^{\mathrm{ph}}\right|_{e_{i}^{P}=0}$. Equation (A9) is just the answer expressed by (26) and (27). 


\section{References}

[1] V. L. Berezinskii, Zh. Eksp. Teor. Fiz. 59 (1970), 907-920

[2] V. L. Berezinskii, Zh. Eksp. Teor. Fiz. 61 (1971), 1144-1156

[3] J. M. Kosterlitz, D. J. Thouless, J. Phys. C: Solid State Phys. 5 (1972), L124-L126

[4] J. M. Kosterlitz, D. J. Thouless, J. Phys. C: Solid State Phys. 6 (1973), 1181-1203

[5] V. N. Popov, Teor. Mat. Fiz. 11 (1972), 354-365

[6] V. N. Popov, Zh. Eksp. Teor. Fiz. 64 (1973), 674-680

[7] A. Holz, J. T. N. Medeiros, Phys. Rev. B 17 (1978), 1161-1174

[8] D. R. Nelson, Phys. Rev. B 18 (1978), 2318-2338

[9] D. R. Nelson, B. I. Halperin, Phys. Rev. B 19 (1979), 2457-2484

[10] A. P. Young, Phys. Rev. B 19 (1979), 1855-1866

[11] D. R. Nelson, Defects in Superfluids, Superconductors, and Membranes, In: "Fluctuating Geometries in Statistical Mechanics and Field Theory", Les Houches Proceedings, Session LXII, 1994 (NATO ASI Series vol 62), 423-478 ArXiv: cond-mat/9502114

[12] H. Kleinert, Gauge Fields in Condensed Matter. Superflow and Vortex Lines. Vol. I (World Scientific, Singapore, 1989)

[13] H. Kleinert, Gauge Fields in Condensed Matter. Stresses and Defects. Vol. II (World Scientific, Singapore, 1989)

[14] H. Kleinert, Vortex, Defect, and Monopole Gauge Fields for Nambu-Goldstone Systems and their Phase Transitions, ArXiv: cond-mat/9503030

[15] T. Yamamoto, T. Izuyama, J. Phys. Soc. Japan 57 (1988), 3742-3752

[16] S. Panyukov, Y. Rabin, Phys. Rev. B 59 (1999-I), 13657-13671

[17] J. Zaanen, Z. Nussinov, S. I. Mukhin, Ann. Phys. (NY) 310 (2004), 181-260

[18] P. D. Ispánovity, I. Groma, G. Györgyi, Phys. Rev. B 78 (2008), 024119 [10 pages]

[19] R. Saito, G. Dresselhaus, M. S. Dresselhaus, Physical Properties of Carbon Nanotubes (Imperial College Press, Imperial College, London, 1998)

[20] D. Tománek, R. J. Enbody (Eds.), Science and Application of Nanotubes (Kluwer Academic Publishers, etc., New York, 2002)

[21] M. Yu. Gutkin, A. G. Sheinerman, Phys. Solid State 49 (2007), 1672-1679

[22] J. Dietel, H. Kleinert, Phys. Rev. B 79 (2009), 245415

[23] A. Carpio, L. L. Bonilla, F. de Juan, M. A. H. Vozmediano, New Journal of Physics 10 (2008), 053021 
[24] F. de Juan, A. Cortijo, M. A. H. Vozmediano, Nucl. Phys. B 828 (2010), 625-637

[25] S. Bhowmick, U. V. Waghmare, Phys. Rev. B 81 (2010), 155416

[26] R. E. Peierls, Proc. Phys. Soc. 52 (1940), 34-37

[27] F. R. N. Nabarro, Proc. Phys. Soc. 59 (1947), 256-272

[28] A. D. Brailsford, Phys. Rev. 142 (1966), 383-387

[29] I. A. Kunin, Theory of Elastic Media with Microstructure (Nauka, Moscow, 1975) [In Russian]

[30] A. C. Eringen, J. Appl. Phys. 54 (1983), 4703-4710

[31] M. Yu. Gutkin, E. C. Aifantis, Scr. Mater. 35 (1996), 1353-1358

[32] M. Yu. Gutkin, E. C. Aifantis, Scr. Mater. 36 (1997), 129-135

[33] M. C. Valsakumar, D. Sahoo, Bull. Mater. Sci. 10 (1988), 3-44

[34] D. G. B. Edelen, Int. J. Engng Sci. 34 (1996), 81-86

[35] C. Malyshev, Ann. Phys. (NY) 286 (2000), 249-277

[36] M. Lazar, J. Phys. A: Math. Gen. 35 (2002), 1983-2004

[37] M. Lazar, J. Phys. A: Math. Gen. 36 (2003), 1415-1438

[38] A. Seeger, The application of second-order effects in elasticity to problems of crystal physics, In: Second-Order Effects in Elasticity, Plasticity, and Fluid Dynamics. Int. Symp., Haifa, Israel, April 23-27, 1962 (Eds., M. Reiner, D. Abir, Pergamon Press, Oxford, 1964), pp. 129-144

[39] C. Malyshev, J. Phys. A: Math. Theor. 40 (2007), 10657-10684

[40] V. N. Popov, Functional Integrals in Quantum Field Theory and Statistical Physics (D. Reidel, Dordrecht,1983)

[41] V. N. Popov, Functional Integrals and Collective Excitations (Cambridge University Press, Cambridge, 1987, 1990)

[42] R. J. Rivers, Path Integral Methods in Quantum Field Theory (Cambridge University Press, Cambridge, 1987)

[43] V. N. Popov, V. S. Yarunin: Collective Effects in Quantum Statistics of Radiation and Matter (Kluwer, Dordrecht, 1988)

[44] H. Kleinert, Path Integrals in Quantum Mechanics, Statistics, Polymer Physics, and Financial Markets (World Scientific, Singapore, 1990, 1995, 2004)

[45] J. Zinn-Justin, Quantum Field Theory and Critical Phenomena (Clarendon Press, Oxford, 1996) 
[46] C. Deutsch, M. Lavaud, Phys. Rev. A 9 (1974), 2598-2616

[47] J. V. José, L. P. Kadanoff, S. Kirkpatrick, D. R. Nelson, Phys. Rev. B 16 (1977), $1217-1241$

[48] B. Nienhuis, J. Stat. Phys. 34 (1984), 731-761

[49] A. M. Polyakov, Gauge Fields and Strings (Harwood, London, 1987)

[50] A. A. Abrikosov (jr), Ya. I. Kogan, Zh. Eksp. Teor. Fiz. 96 (1989), 418-436

[51] A. A. Abrikosov (jr), Ya. I. Kogan, Int. J. Mod. Phys. A 6 (1991), 1501-1524

[52] C. Teodosiu, Elastic Models of Crystal Defects (Springer-Verlag, Berlin, etc., 1982)

[53] J. P. Hirth, J. Lothe, Theory of Dislocations (Wiley, New York, etc., 1982)

[54] M. O. Katanaev, I. V. Volovich, Ann. Phys. (NY) 216 (1992), 1-28

[55] G. Sardanashvily, Theor. Math. Phys. 132 (2002), 1163-1171

[56] F. W. Hehl, Y. N. Obukhov, Ann. Fond. L. de Broglie 32 (2007), 157-194

[57] G. de Berredo-Peixoto, M. O. Katanaev, Tube Dislocations in Gravity, ArXiv: 0810.0243

[58] H. Kleinert, J. Zaanen, Phys. Lett. A 324 (2004), 361-365

[59] R. de Wit, Linear theory of static disclinations, In: Fundamental Aspects of Dislocation, Nat. Bur. Stand. (US), Spec. Publ. 317, vol. 1, 1970 (Eds., J. A. Simmons, R. de Wit, R. Bullough), pp. 651-673

[60] R. de Wit, J. Res. Natl. Bur. Stand. Sect. A 77 (1973), 49-100; 359-368; 607-658

[61] E. Kröner, Continuum theory of defects, In: Physique des Défauts, Les Houches, Session XXXV, 1980 (Eds., R. Balian, et al., North-Holland, Amsterdam, 1981), pp. $215-316$

[62] M. Lazar, G. A. Maugin, E. C. Aifantis, Phys. Stat. Sol. (b) 242 (2005), 2365-2390

[63] S. Coleman, Phys. Rev. D 11 (1975), 2088-2097

[64] S. Samuel, Phys. Rev. D 18 (1978), 1916-1932

[65] J. Fröhlich, T. Spencer, J. Stat. Phys. 24 (1981), 617-701

[66] L. Mondaini, E. C. Marino, J. Stat. Phys. 118 (2005), 767-779 